\title{
Variability in amplitude of untreated essential tremor
}

\author{
LYNN CLEEVES, LESLIE J FINDLEY \\ From the MRC Neuro-Otology Unit, National Hospital for Nervous Diseases, London, and the Regional Centre \\ for Neurology and Neurosurgery, Oldchurch Hospital, Romford, UK
}

SUMMARY Tremor amplitude was monitored in untreated patients with essential tremor by a procedure designed to minimise fluctuations due to factors known to influence tremor. Tremor was measured hourly for 6 hours in 14 patients and on five to seven occasions (separated by at least a week) in 22 patients. A wide range of within-patient variability was found, with some patients showing little and others showing considerable fluctuations in amplitude over the series of recordings. There was no simple relationship between variability and mean amplitude, frequency, age, duration of tremor or response to a single oral dose of propranolol. Variability did not differ between males and females or between patients with and without a family history of tremor. Diurnal fluctuations showed no consistent pattern. Long-term assessment revealed a significant trend towards lower amplitudes on successive occasions. To overcome systematic bias in data collection, clinical trials in essential tremor should employ large numbers of patients and rigorous randomisation of treatments in cross-over designs.

At onset essential tremor typically affects the hands and is of low amplitude. Initially it may be intermittent, though when fully developed it manifests as continuous tremoi of the hands in posture and during movement. Essential tremor can occur at any age and is usually slowly progressive; in younger patients an initial increase in severity in the first few years may be followed by a "plateau" of relative stability with a further progression in later life, ${ }^{12}$ often leading to considerable functional impairment.

As well as a gradual progression with age, it is well known that tremor amplitude can be influenced in the short-term by a number of physiological and psychological factors. For example, cold, hunger, muscular fatigue, anxiety and stressful social situations commonly exacerbate essential tremor. ${ }^{2}$ In many patients a small quantity of alcohol can rapidly and markedly attenuate tremor amplitude, ${ }^{34}$ though a rebound increase in tremor may occur as the blood alcohol level falls. ${ }^{5}$ Smoking may exacerbate tremor in some patients and attenuate it in others. ${ }^{2}$

Fluctuations of tremor amplitude in untreated patients clearly have a considerable bearing on evalu-

Address for reprint requests: Dr L Cleeves, MRE Neuro-Otology Unit, National Hospital for Nervous Diseases, Queen Square, London WCIN 3BG, UK

Received 12 August 1986. Accepted 27 September 1986 ation of the efficacy of tremorlytic drugs. Most clin ical trials in essential tremor have attempted to cong trol for the most obvious sources of extraneous variation ${ }^{67}$ and it is generally accepted that, in the absence of such extraneous factors, the amplitude of the "underlying" essential tremor is not likely to varyo appreciably over relatively short periods of time, for example, over the course of a clinical trial lasting several weeks or months. Certainly, it is rare to observe in essential tremor the sudden and extreme fluctuations in amplitude from moment to moment seen in the classical resting tremor of Parkinson's disease, a phenomenon which has bedevilled attempts to monitor the tremorlytic effects of antiparkinsonian medication. ${ }^{8}$

The purpose of the present study was to assess the variability of tremor amplitude in untreated patients with essential tremor in a strictly controlled laboratory environment designed to minimise fluctuations due to identified extraneous factors.

\section{Patients and methods}

Thirty six patients with essential tremor of the hands participated in the study (table 1). Diagnosis was made on the basis of clinical history and detailed general and neurological examination. Serum triodothyronine and thyroxine levels were within normal limits in all cases. Patients with a history of excessive alcohol consumption were excluded. Only two patients had previously taken medication for 
Table 1 Patient data

\begin{tabular}{|c|c|c|}
\hline & Diurnal study & Long-term study \\
\hline $\begin{array}{l}\text { Number of patients } \\
\text { Sex } \\
\text { Family history of tremor* } \\
\text { Age (yr) }\end{array}$ & $\begin{array}{l}14 \\
4(\mathrm{~F}) 10(\mathrm{M}) \\
6(+) 7(-) \\
35-79 \\
\quad(\text { mean 59.4) }\end{array}$ & $\begin{array}{l}22 \\
7(\mathrm{~F}) 15(\mathrm{M}) \\
8(+) 12(-) \\
17-73 \\
\quad \text { (mean 49.1) }\end{array}$ \\
\hline Duration (yr) & $\begin{array}{l}1-50 \\
(\text { mean } 12 \cdot 2)\end{array}$ & $\begin{array}{l}1-50 \\
(\text { mean } 14 \cdot 1)\end{array}$ \\
\hline Magnitude $\left(g \times 10^{-3}\right)$ & $\begin{array}{c}5.3-310.8 \\
(\text { mean } 39.5)\end{array}$ & $\begin{array}{l}4 \cdot 2-289 \cdot 0 \\
(\text { mean } 29 \cdot 1)\end{array}$ \\
\hline Peak frequency $(\mathrm{Hz})$ & $\begin{array}{l}4 \cdot 8-7 \cdot 2 \\
(\text { mean } 6 \cdot 1)\end{array}$ & $\begin{array}{l}4 \cdot 1-9 \cdot 8 \\
\quad(\text { mean } 6 \cdot 5)\end{array}$ \\
\hline
\end{tabular}

*Family history unknown in 1 patient in diurnal group and 2 in long-term group.

tremor (propranolol $240 \mathrm{mg}$ and $120 \mathrm{mg}$ daily respectively) which was withdrawn 2 weeks prior to the study. Patients were requested to abstain from caffeinated beverages, alcohol and tobacco for at least 12 hours prior to assessment.

Fourteen patients attended for a single day during which tremor was measured accelerometrically (see below) at
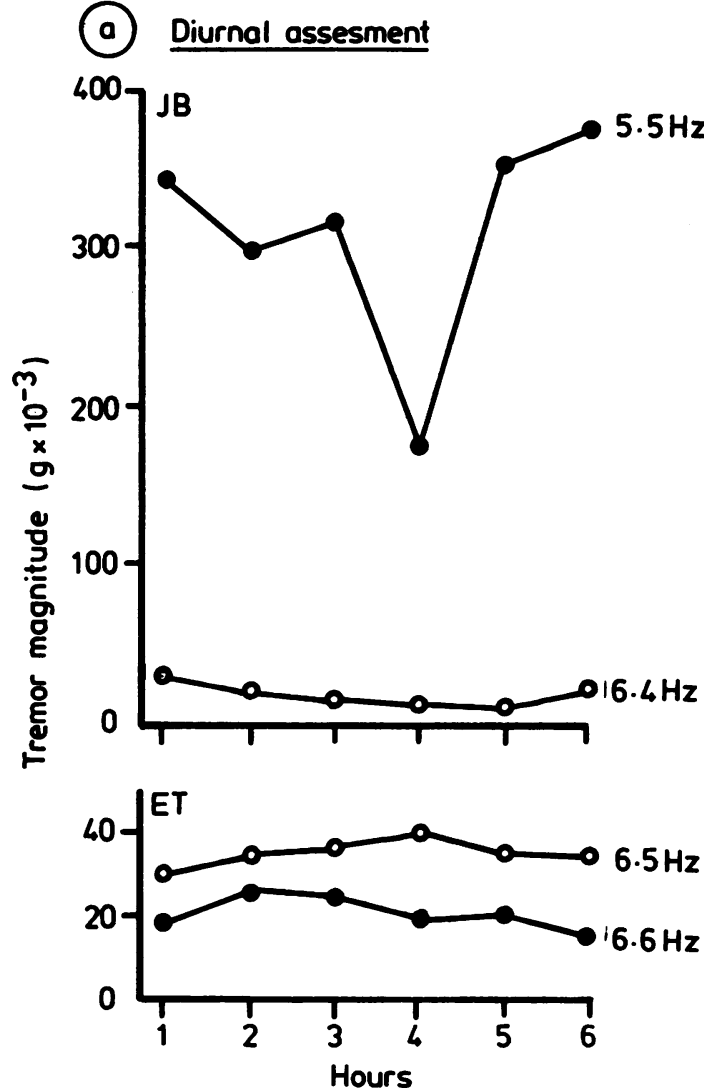

hourly intervals for 6 hours. Patients were requested not to leave the hospital between recordings and to relax in a quiet room for at least 15 minutes prior to each recording. A light lunch was taken between the third and fourth recordings. A further 22 patients attended for single assessments on five to seven occasions at intervals of not less than one week. One patient attended for two series of six weekly recordings separated by a period of 28 weeks. Patients were given 30 minutes relaxation prior to assessments which were always carried out at the same time of day.

Tremor measurement The patient was seated with the forearms supported up to the wrists. Miniature piezo-resistive linear accelerometers (Endevco 7625-10) were attached to the dorsal surface of each hand in the second interspace, one $\mathrm{cm}$ proximal to the metacarpophalangeal joints. The devices weighed $6 \mathrm{~g}$ and had a frequency response extending from steady state acceleration to $300 \mathrm{~Hz}$ with a sensitivity of $50 \mathrm{mV} / g$ ( $g=$ acceleration of gravity). Tremor was measured with the hands outstretched horizontally in pronated posture. Three separate tremor recordings of about $1 \mathrm{~min}$ duration were made at $3 \mathrm{~min}$ intervals. To minimise fatigue, the hands were allowed to relax freely between recordings. Accelerometric signals were amplified and analysed on-line
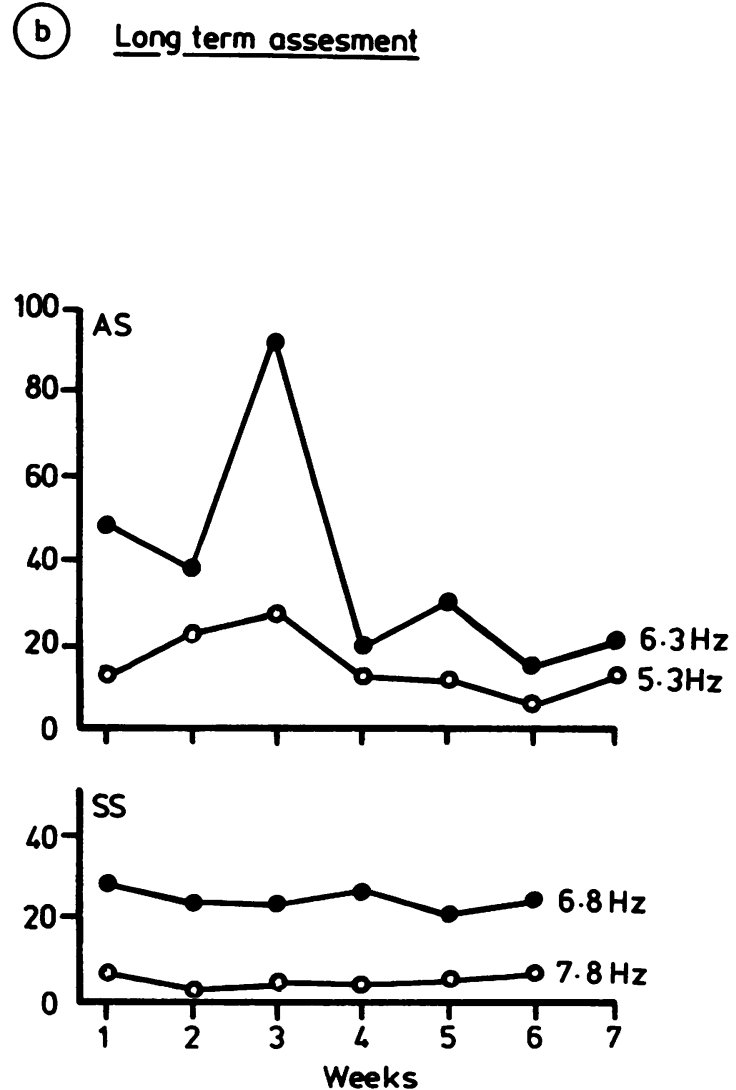

Fig 1 Examples of tremor magnitudes recorded in individual patients over consecutive diurnal (A) and long-term (B) assessments, showing considerable (upper graphs) or little (lower graphs) variability. (filled circles, right hand; open circles, left hand). 
Table 2 Results of linear regression analysis showing correlation coefficient $(r)$ and $F$-ratio (variance due to regression/residual variance). No values reach statistical significance at $5 \%$ level

\begin{tabular}{lll}
\hline & Long-term study & Diurnal study \\
\hline CV on frequency & $\mathrm{r}=-0.16 ; \mathrm{F}=1.06^{*}$ & $\mathrm{r}=+0.31 ; \mathrm{F}=2.74 \dagger$ \\
Log magnitude & $\mathrm{r}=+0.07 ; \mathrm{F}=0.23$ & $\mathrm{r}=-0.02 ; \mathrm{F}=0.03$ \\
$\begin{array}{l}\text { Age } \\
\text { Log duration }\end{array}$ & $\mathrm{r}=+0.05 ; \mathrm{F}=0.13$ & $\mathrm{r}=+0.17 ; \mathrm{F}=0.83$ \\
$\begin{array}{l}\text { Response to } \\
\text { propranolol }\end{array}$ & $\mathrm{r}=+0.27 ; \mathrm{F}=1.43$ & $\mathrm{r}=-0.01 ; \mathrm{F}=0.004$ \\
\hline
\end{tabular}

*df 1,42 . tdf 1,26 .

using a Hewlett Packard 5420A signal analyser. ${ }^{9}$ The program averaged 150 autospectra each derived from $10.24 \mathrm{sec}-$ ond samples of tremor. Fifty samples were taken from each of the three separate recording periods and approximately 45 seconds of tremor recording contributed to the analysis in each period. The averaged spectra were displayed as root mean square (rms) magnitude of the frequency components plotted as a function of frequency. Measurements were taken of the frequency $(\mathrm{Hz})$ of the dominant peak and of its amplitude scaled in rms acceleration, the unit of acceleration being taken as $\lg \left(g=981 \mathrm{~cm} / \mathrm{s}^{2}\right)$.

\section{Results}

Magnitude variability Mean tremor magnitudes of the patients in the two study groups are presented in table 1. Variability of magnitude within patients is expressed as the coefficient of variation (CV) that is, the standard deviation of the series of tremor measurements for an individual patient expressed as a proportion of the mean value (SD/Mean). The $\mathrm{CV}$ was used as it is independent of absolute tremor magnitude and takes every score into account. CVs were computed separately for each hand, thus in statistical analyses $n=28$ (diurnal) and $n=44$ (long-term).

A wide range of variability in tremor magnitudes was observed for both diurnal and long-term recordings with some patients showing little change in magnitude from one recording to another and others showing considerable changes (fig 1). CVs ranged from 0.09 to 0.87 (mean 0.32 ) in the diurnal group and from 0.08 to 0.94 (mean 0.43 ) in the long-term group. Variability was generally lower in the diurnal group (the mean CV for this group is distorted by a

Table 3 Coefficient of variation (mean $\pm S E M$ ) for males and females and patients with and without a family history of tremor

\begin{tabular}{lll}
\hline & Diurnal study & Long-term study \\
\hline Male & $33.3 \pm 4.0$ & $44.6 \pm 3.3$ \\
Female & $27.4 \pm 4.4$ & $40.1 \pm 6.0$ \\
Positive F.H. & $33.1 \pm 5.5$ & $41.9 \pm 6.0$ \\
Negative F.H. & $27.8 \pm 3.8$ & $45.2 \pm 3.6$ \\
\hline
\end{tabular}

single high value of 0.87 , the next highest value being 0.52 ).

To ascertain whether magnitude variability in either group could be predicted by some other variable, linear regression analyses were performed (data for duration and mean magnitude were logarithmically transformed to normalise their distributions). Results (table 2) did not reveal any simple
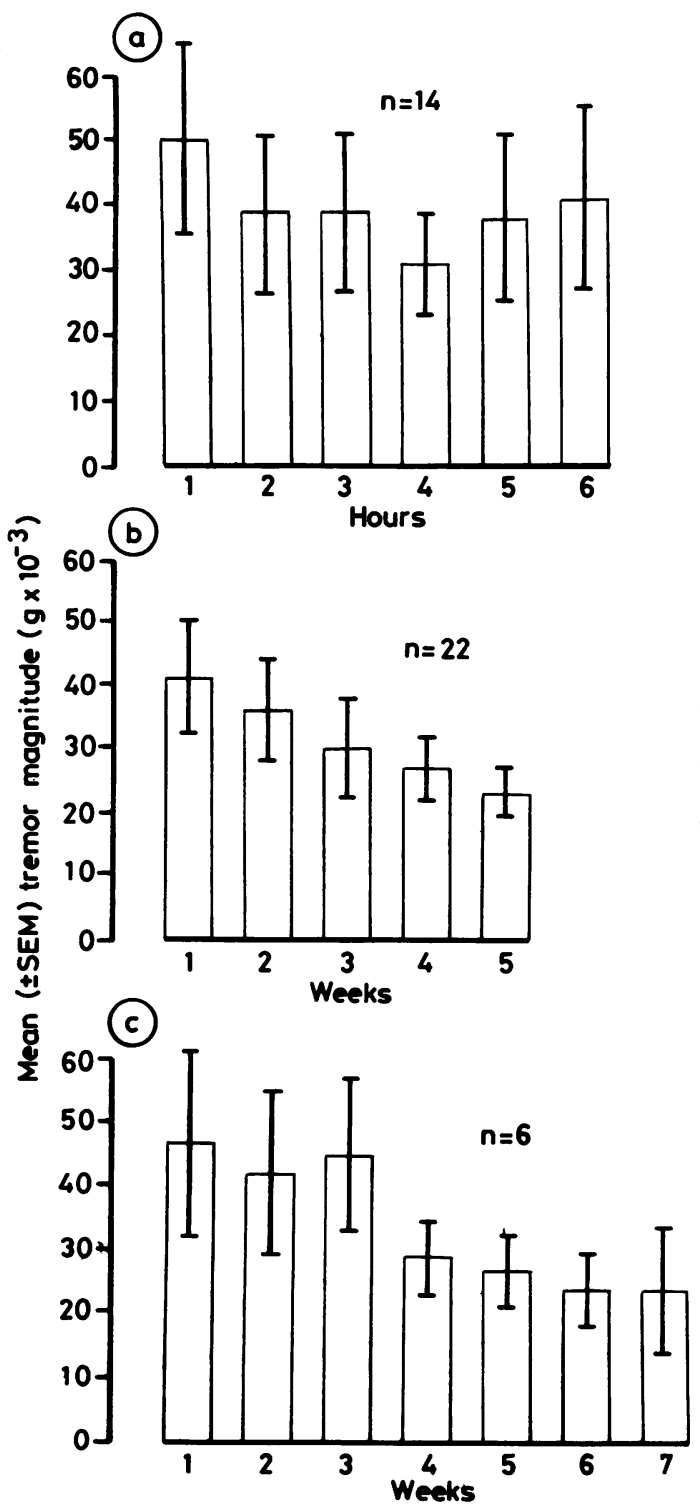

Fig 2 Mean tremor magnitudes ( $\pm S E M)$ for consecutive assessments. (A) Diurnal study, (B) long-term study, (C) sub-group of 6 patients in long-term study attending for seven assessments. 
linear relationship between degree of variability (CV) and age, log duration of tremor, log mean tremor magnitude and mean frequency, or (long-term group only) between variability and response to a single oral dose $(120 \mathrm{mg})$ of propranolol assessed by the same laboratory procedure. There were no differences in variability of tremor magnitude between males and females or between patients with and without a family history of tremor (table 3 ).

Observation of the data suggested a trend towards a reduction in tremor amplitude with successive assessments in some patients, particularly in the longterm group (for example, fig 1B, upper graph). Further analysis of the data was therefore carried out to determine if the observed fluctuations were random or whether there was some systematic variation over time. Group means of tremor magnitude were computed for successive assessments (first five assessments in the long term group) (fig 2A, B). Analysis of variance for repeated measures showed no difference in mean tremor levels over 6-hourly assessments in the diurnal group. There was however, a significant difference $(F=4.36$, df $4,172, p<0.01)$ between mean tremor levels recorded in the long-term group, with a significant linear trend $(p<0.01)$. Pairwise comparisons of means (Newman-Keuls test) indicated a significant difference in tremor levels between assessments 5 and 1 (p $<0.01), 5$ and $2(p<0.05)$, and between 4 and $1(p<0.05)$.

Frequency variability Mean frequency of tremor for patients in the two groups is presented in table $1 . \mathrm{CVs}$ for frequency ranged from 0.001 to 0.09 , mean 0.04 (diurnal) and from 0.01 to 0.22 , mean 0.05 (long term). In terms of absolute units, frequency varied by less than $1 \mathrm{~Hz}$ in the majority of patients $(78.6 \%$ in the diurnal group, $65.9 \%$ in the long term group) and by less than $0.5 \mathrm{~Hz}$ in $39.3 \%$ and $47.7 \%$ respectively.

\section{Discussion}

The results indicate that under identical conditions of assessment and when major factors known to influence tremor amplitude are minimised, considerable fluctuations in the amplitude of untreated essential tremor can be observed. Clinical rating of tremor was not specifically performed in this study. However, the variations in amplitude measured accelerometrically were often associated with clinically observable changes. Though a few patients showed remarkably consistent amplitudes over the study period, 17 of 22 patients in the long-term group had CVs of 0.25 or greater (that is, standard deviation at least $25 \%$ of mean value). Variability was generally less in the diurnal group with only five of 14 patients having CVs of 0.25 or greater. Variability was not related to any other factor investigated, that is, age, sex, duration, family history, mean magnitude or frequency of tremor.

In contrast to the wide range of magnitude variability, frequencies were remarkably consistent. Frequency typically varied by less than $1 \mathrm{~Hz}$ and in some patients was almost constant with as little as $0.1 \mathrm{~Hz}$ variation over the series of recordings. Tremors showing such "invariant" frequency could be of high or low frequency and high or low amplitude. However, widely variable peak frequency (up to $2 \mathrm{~Hz}$ in some patients) tended to be associated with moderate to low amplitude tremors of higher frequency where multiple peaks appeared in the tremor spectrum.

The cause of the observed fluctuations in amplitude is unknown. However, the results suggest that for purposes of design and data analysis in clinical trials, diurnal fluctuations can be considered to be random since there was no consistent pattern of variability between patients and no differences in group means of tremor magnitude over successive assessments. This was not the case, however, for changes in amplitude recorded over longer periods of time. A systematic and statistically significant decrement in mean amplitude was found over five consecutive assessments carried out at approximately weekly intervals. To gain some idea of how long this decline might continue, consecutive mean amplitudes were computed for a sub-group of six patients in the long-term group who attended for a total of seven assessments. Mean tremor levels for this group appear to stabilise at around 4 to 5 weeks (fig $2 \mathrm{C}$ ).

The nature of this systematic decline in tremor amplitudes is not clear. The time course would seem to suggest some factor other than simple anxiety reduction. Whilst most patients admitted to some degree of apprehension on the first visit, all claimed to be perfectly at ease on subsequent visits. The effect does not appear to be related simply to familiarisation due to repeated assessments since there was no such decline in the diurnal group over six assessments. One patient who attended for two series of six weekly assessments separated by an interval of 28 weeks showed a higher level of tremor at the beginning of the second series than at the end of the first with a subsequent decline mirroring that seen in the first series. This pattern of response decrement with recovery over time is reminiscent of the phenomenon of habituation seen in a wide variety of behavioural situations. ${ }^{10}$

Whatever its cause, a systematic decline in tremor amplitudes over a number of assessments has implications for the design of clinical trials in essential tremor. Cross-over trials have the advantage of within-subject comparison of treatment effects and permit the use of considerably - smaller numbers of patients than independent groups designs. ${ }^{11}$ The 
cross-over design assumes, however, that the state of the patient does not differ substantially from one treatment period to the next, either as a result of the treatment or changes in the disease state. The systematic decrease in tremor amplitude observed in the present study resulted in statistically significant differences between means when later assessments were compared with earlier assessments (see Results). Mean tremor levels for any two adjacent assessments were not significantly different. Thus, trials comparing two or three treatments, with strictly randomised allocation of patients to each, should not fall foul of systematic bias provided a sufficiently large number of patients is employed. Small numbers of patients may not be adequate to overcome this bias even with randomised order of treatments.

In the present study, by drawing sub-samples of eight patients from the larger group of 22 and randomly allocating their week 1 and week 5 data to two hypothetical "treatment" groups according to a cross-over design, we have been able to demonstrate statistically significant differences between "treatments". When data from an additional four patients were included, these significant differences disappeared. "Cross-over" analysis of data from the entire group of 22 resulted in almost identical "treatment" means. Thus, on an empirical basis we would advocate sample sizes of at least 12 patients in long-term cross over trials in essential tremor.

Bias from the sequential changes in tremor amplitude reported in the present study could engender both false positives (type 1 error) and false negatives (type 2 error) in data analysis when small numbers of patients are used. Several studies have appeared in the literature in which propranolol was found to be no different from placebo. ${ }^{12-14}$ In all these studies, the lack of efficacy may have been due to the dose of propanolol used (60-120 mg daily). In double-blind, controlled clinical trials, ${ }^{1516} 120 \mathrm{mg}$ propranolol has been shown to be effective in reducing the amplitude of essential tremor when given as a single dose but not when given as a divided daily dose. However, it is interesting to note that Balla ${ }^{12}$ using seven patients and weekly assessments remarked that patients tended "to improve, particularly towards the third and fourth week of treatment. This was irrespective of whether placebo or active drugs were used at that stage". Thus, a tendency towards tremor reduction with repeated assessments, as found in the present study, may have obscured the effect of propranolol.

\section{References}

1 Critchley M. Observations on essential (heredofamilial) tremor. Brain 1949;72:113-39.

2 Rautakorpi I. Essential tremor. An epidemiological, clinical and genetic study. Res Rep Dept Neurol, Univ Turku, Finland 1978; No 12.

3 Rajput AH, Jamieson H, Hirsch S. Specificity of tremorlytic effects of alcohol and propranolol. Clin Res 1973;21:1018.

4 Murray TJ. Essential tremor. Can Med Assoc J 1981;124:1559-65.

5 Growdon JH, Shahani BT, Young RR. The effect of alcohol on essential tremor. Neurology 1975;25:259-62.

6 Winkler GF, Young RR. The control of essential tremor by propranolol. Trans Am Neurol Assoc 1971;96:66-8.

7 Cleeves L, Findley LJ. Beta-adrenoreceptor mechanisms in essential tremor: a comparative single dose study of the effect of a non-selective and a beta-2 selective adrenoreceptor antagonist. J Neurol Neurosurg Psychiatry 1984;47:976-82.

8 Cleeves L, Findley LJ, Gresty MA. Assessment of rest tremor in Parkinson's disease. In: Yahr MD, Bergmann KJ, eds. Advances in Neurology. Vol 45. New York: Raven Press, 1986:349-52.

9 Hewlett-Packard. Digital Signal Analyser: User's Guide. HP 05420-900, 1978.

10 Thompson RF, Spencer WA. Habituation: A model phenomenon for the study of neuronal substrates of behaviour. Psychol Rev 1966;73:16-43.

11 Feinstein AR. Clinical Biostatistics. St Louis: C.V.co Mosby Co., 1977:320-34.

12 Balla JI. Treatment of essential tremor with propranolol. Lancet 1973;1:205.

13 Foster JB, Longley BP, Stewart-Wynne EG. Propranolol in essential tremor. Lancet 1973;1:1455.

14 Sweet RD, Blumberg J, Lee JE, McDowell MC. Propranolol treatment of essential tremor. Neurology 1974;24:64-7.

15 Calzetti S, Findley LJ, Gresty MA, Perucca E, Richens A. Effect of a single dose of propranolol on essential tremor: A double-blind controlled study. Ann Neurol 1983;13:165-71.

16 Calzetti S, Findley LJ, Perucca E, Richens A. The response of essential tremor to propranolol: evaluation of clinical variables governing its efficacy on prolonged administration. J Neurol Neurosurg Psychiatry 1983;46:393-8. 